\title{
LOCAL LEFT NOETHERIAN IPLI-RINGS
}

\author{
BY ARUN VINAYAK JATEGAONKAR
}

Communicated by I. N. Herstein, September 30, 1968

All rings are associative and unitary. A ring $R$ is a pli-ring (resp. ipli-ring) if every left ideal (resp. two-sided ideal) of $R$ is of the form $R a$ for some $a \in R$. Clearly, every pli-ring is a left Noetherian ipli-ring. A ring $R$ is called local if $R$ has a unique maximal left ideal.

This note contains statements of some results concerning ideals and global dimensions of local left Noetherian ipli-rings.

A few definitions are needed. Let $I$ be an ideal (i.e., two-sided ideal) of a ring $R$. We shall give two definitions of transfinite powers of $I$. The first is: $I^{1}=I ; I^{\alpha}=I \cdot I^{\beta}$ if $\alpha=\beta+1 ; I^{\alpha}=\bigcap_{\beta<\alpha} I^{\beta}$ if $\alpha$ is a limit ordinal. The second definition is notationally distinguished from the first by writing the index ordinal in a square bracket; it goes as follows:

$$
I^{\left[\omega^{0}\right]}=I ; \quad I^{[\omega \alpha]}=\bigcap_{n=1}^{\infty}\left(I^{[\omega \beta]}\right)^{n} \quad \text { if } \alpha=\beta+1 ; \quad I^{[\omega \alpha]}=\bigcap_{\beta<\alpha} I^{[\omega \beta]}
$$

if $\alpha$ is a limit ordinal. Note that the second definition defines transfinite powers only for ordinals of the form $\omega^{\alpha}$. For all the set-theory involved, we refer to [3].

The following theorem is basic.

THEOREM 1. Let $A$ be a proper prime ideal in a prime left Noetherian ipli-ring $R$. Then there exists an ordinal $\alpha$ such that $A^{\left[\omega^{\alpha}\right]}=(0)$. Let $\alpha$ be the first such ordinal. Then $A^{\left[\omega^{\beta}\right]} \overbrace{}^{\left[\omega^{\beta}\right]}$ if $\gamma<\beta \leqq \alpha$. The prime ideals of $R$ contained in $A$ are precisely those of the form $A^{\left[\omega \omega^{\beta}\right]}$ where $\beta \leqq \alpha$.

Recall that a domain is a (not necessarily commutative) ring without zero-divisors.

THEOREM 2. Let $R$ be a local semiprime left Noetherian ipli-ring with Jacobson radical $J$. Then

(1) $R$ is a pli-domain.

(2) There exists an ordinal $\alpha$ such that $J^{\left[\omega^{\alpha}\right]}=(0)$. Let $\alpha$ be the first such ordinal. For every $\beta<\alpha$, choose $x_{\beta} \in R$ such that $J^{\left[\omega^{\beta}\right]}=R x_{\beta}$.

(3) Every nonzero element $r$ of $R$ can be uniquely expressed as

$$
r=u x_{\beta_{1}}^{m_{1}} \cdots x_{\beta_{1}}^{m_{1}},
$$

where $s$ is a nonnegative integer, $m_{i} \in Z^{+}, \beta_{1}<\cdots<\beta_{0} \leqq \alpha$ and $u$ is a unit in $R$. 
(4) Every left ideal of $R$ is two-sided. Ideals form a well-ordered set under reverse inclusion. Every nonzero ideal $A$ can be uniquely written as

$$
A=\left(R x_{\beta_{1}}\right)^{m_{1}} \cdots\left(R x_{\beta_{d}}\right)^{m_{a}},
$$

where $s$ is a nonnegative integer, $m_{i} \in Z^{+}$and $\beta_{1}<\cdots<\beta_{\text {s }} \leqq \alpha$. Further,

$$
A \mapsto \omega^{\beta} m_{0}+\cdots+\omega^{\beta_{1} m_{1}}
$$

is an order-isomorphism of the well-ordered set of all nonzero ideals of $R$ under reverse inclusion and the set of all ordinals $<\omega^{\alpha}$. The same bijection is an anti-isomorphism of the monoid of all nonzero ideals of $R$ under the usual multiplication and the set of all ordinals $<\omega^{\alpha}$ under the usual addition.

(5) Every ideal of $R$ is of the form $J^{\lambda}$ for some uniquely determined $\lambda \leqq \omega^{\alpha}$. If the Cantor normal form of $\lambda$ is

$$
\lambda=\omega^{\beta_{2} m_{t}}+\cdots+\omega^{\beta_{1} m_{1}}
$$

then $J^{\lambda}=\left(R x_{\beta_{1}}\right)^{m 1} \cdots\left(R x_{\beta_{0}}\right)^{m_{0}}$. In particular, $J^{\omega \beta}=J^{\left(\omega_{1}\right]}$ for every $\beta \leqq \alpha$.

Recall that a ring $R$ is completely primary if $R / P(R)$ is a domain, where $P(R)$ is the prime radical of $R$.

Theorem 3. Let $R$ be a local left Noetherian ipli-ring. Then $R$ is a completely primary pli-ring, every left ideal of $R$ is a two-sided ideal and the set of ideals of $R$ is a well-ordered set under reverse inclusion.

$R$ is a domain if and only if the well-ordered set of nonzero ideals of $R$ under reverse inclusion is order-isomorphic with the set of all ordinals $<\omega^{\alpha}$ for some ordinal $\alpha$.

More details about these rings (similar to Theorem 2) are obtained.

THEOREM 4. Let $A$ be a proper prime ideal in a prime left Noetherian ipli-ring $R$ and let $\lambda$ be an ordinal such that $A^{\left[\omega^{\lambda}\right]} \neq(0)$. Then there exists a set of principal right ideals of $R$ which form a well-ordered set under inclusion order-isomorphic with the set of all ordinals $<\lambda$.

Theorem 4 and a theorem of B. L. Osofsky [2] imply the following.

TheOREM 5. Let $A$ be a proper prime ideal in a left Noetherian iplidomain $R$ and let $\lambda$ be an ordinal such that $A^{\left[\omega^{\lambda}\right]} \neq(0)$. Then

$$
\begin{aligned}
\text { r. } g \text { l. } \operatorname{dim} R & =\infty & & \text { if } \operatorname{card} \lambda \geqq \aleph_{\infty}, \\
& \geqq n+2 & & \text { if } \operatorname{card} \lambda \geqq \aleph_{n}, 0 \leqq n<\omega, \\
& \geqq 2 & & \text { if } \operatorname{card} \lambda \text { is a nonzero integer. }
\end{aligned}
$$


TheOREM 6. Let $A$ be a proper prime ideal in a pli-domain $R$ and let $\lambda$ be an ordinal such that $A^{\left[\omega^{\lambda_{1}}\right.} \neq(0)$. If $\operatorname{card} \lambda=\operatorname{card} R=\aleph_{n}$ where $n$ is a nonzero integer then

$$
\text { r. gl. } \operatorname{dim} R=n+1 ; \quad \text { l. gl. } \operatorname{dim} R=1 \text {. }
$$

If $\operatorname{card} \lambda \geqq \boldsymbol{N}_{\omega}$, then

$$
\text { r. gl. } \operatorname{dim} R=\infty ; \quad \text { 1. gl. } \operatorname{dim} R=1 \text {. }
$$

TheOREM 7 (CF. [1]). Let $1 \leqq m \leqq n \leqq \infty$. Then there exists a left Noetherian domain $D$ such that

$$
\text { r. gl. } \operatorname{dim} D=n ; \quad \text { l. gl. } \operatorname{dim} D=m \text {. }
$$

Acknowledgements. Dr. H. Brungs has obtained the following special case of our results: If left ideals of a ring are well-ordered under reverse inclusion, then (1) they are principal left ideals and (2) they are two-sided. With the same hypothesis, he has obtained the last part of Theorem 3. Statements of Dr. Brungs' results were communicated to the author by Professor P. M. Cohn.

The author is indebted to Professor N. Greenleaf for encouragement.

\section{REFERENCES}

1. A. V. Jategaonkar, A counterexample in ring theory and homological algebra, Notices Amer. Math. Soc. 14 (1967), 660.

2. B. L. Osofsky, Homological dimension and the continuum hypothesis, Trans. Amer. Math. Soc. 132 (1968), 217-230.

3. W. Sierpinski, Cardinal and ordinal numbers, PWN, Warsaw, 1958.

UNIVERSITY OF ROCHESTER, ROCHESTER, NEW YoRK 14627 AND Cornell University, IthacA, New York 14850 